\title{
Quasi-TM MoL/MoM Approach for Computing the Transmission-Line Parameters of Lossy Lines
}

\author{
Gonzalo Plaza, Ricardo Marqués, Member, IEEE, and Francisco Medina, Senior Member, IEEE
}

\begin{abstract}
This paper presents a quasi-TM approach for the fundamental mode of transmission lines with semiconductor substrates and nonperfect metallic conductors. The approach has allowed us to develop a transmission-line model by properly defining frequency-dependent parameters in terms of the quasi-static electric potential and the electric current density along the propagation direction in the line. The previous quasi-TM analysis avoids the involved numerical root finding process typical in full-wave analysis, and overcomes the limitations of the conventional quasi-TEM approach to account for the effects of the longitudinal currents present both in the lossy substrates and in the nonperfect conductors. The transmission-line parameters have been computed by a hybrid technique that combines the method of lines with the method of the moments (MoM). The total CPU effort has been considerably reduced thanks to the possibility of finding closed-form expressions for the reaction integrals appearing in the MoM. Comparisons with previous computed and measured results show the validity of the present model.
\end{abstract}

Index Terms-Conductor losses, coplanar waveguide (CPW), metal-insulator-semiconductor (MIS), method of lines (MoL), microstrip, substrate losses, transmission-line model.

\section{INTRODUCTION}

$\mathbf{I}$ $\mathrm{N}$ RECENT years, the development of monolithic microwave integrated circuits (MMICs) for microwave applications, as well as the increase of the operating frequencies in high-speed very large scale integration (VLSI) circuits has aroused a great interest in the study of electromagnetic propagation in planar transmission lines with semiconductor substrates. In MMICs, these lines can be used, for example, to design filters, power dividers, and electronically controlled phase shifters. In modern high-speed VLSI circuits, the lumped capacitance model for on-chip interconnects is no longer valid for propagation delay times below $100 \mathrm{ps}$ [1], which requires a planar transmission-line model for an accurate design of the devices. In this way, a great effort has been devoted to analyze metal-insulator-semiconductor (MIS) transmission lines, especially microstrip and coplanar waveguides (CPWs) as the most practical structures. The main propagation features of the fundamental mode in MIS lines were analyzed in [2] and [3]. In these papers, the nature of the fundamental mode as a function of the semiconductor resistivity and operating

Manuscript received January 30, 2005; revised September 9, 2005. This work was supported by the Spanish Ministry of Education and Science and by FEDER funds under Project TEC2004-04249-C02-02 and Project TEC2004-03214.

G. Plaza is with the Department of Applied Physics I, University of Seville, 41012 Seville, Spain (e-mail: plaza@us.es).

R. Marqués and F. Medina are with the Department of Electronics and Electromagnetism, University of Seville, 41012 Seville, Spain (e-mail: marques@us.es; medina@us.es).

Digital Object Identifier 10.1109/TMTT.2005.860507 frequency (dielectric, slow-wave, and skin-effect modes) is described using a parallel-plate model. Further analysis of MIS transmission lines using a full-wave analysis have been carried out using, for instance, mode-matching techniques, [4], [5], the method of lines (MoL) [6], and the spectral-domain approach (SDA) [7], [8]. More complete full-wave analysis including conductor losses have been carried out by the MoL [9], [10] or by means of discretization techniques such as the finite-difference time-domain (FDTD) method [11], and finite-element method (FEM) [12], [13]. A great number of models based on quasi-static approaches can also be found in the literature. For example, a MIS microstrip line with a zero thickness perfect strip has been analyzed in [14] using a nonuniform cross-sectional transverse resonance technique, and in [15] by means of a quasi-TM SDA. Conductor losses have been also incorporated in the quasi-static approach in [16], where a simple model for the slow-wave zone in a MIS CPW is presented, and in [17], where single and coupled MIS microstrip lines are analyzed by the method of the filaments.

This paper will focus on the computation of the propagation constant and the characteristic impedance of the fundamental mode in planar transmission lines with nonperfect conductors printed on layered dielectric and/or semiconductor substrates. The analysis will follow the quasi-TM approach reported in [15] and will be extended to include the nontrivial case of nonperfect metallic thick conductors. The inclusion of conductor losses is of special importance from a practical point-of-view considering that they can dominate the overall losses even at relatively high frequencies if narrow strip are used [13], [16]. From the quasi-TM approach, a transmission-line model has been developed by properly defining the line voltage and current, as well as the per unit length (p.u.l.) shunt complex capacitance and the p.u.l. series complex inductance. It is important to mention that all the above definitions were carefully carried out to be compatible with the usual concept of complex power, as required by the circuit modeling techniques [18]. As a consequence, the usual expressions for the phase constant and the characteristics impedance in terms of the transmission-line parameters, as well as the usual expressions relating the voltage, current, and characteristic impedance with the complex power remain valid.

Under this approach, the computation of the transmission-line parameters requires to compute the quasi-static electric potential and the longitudinal electric current density in the line. For this purpose, the integral equation for the longitudinal current density in the line will be solved by means of a hybrid technique that combines the MoL (to previously compute the quasi-static electric potential and spatial Green's function for the magnetic potential) with the method of moments (MoM) (to finally obtain 


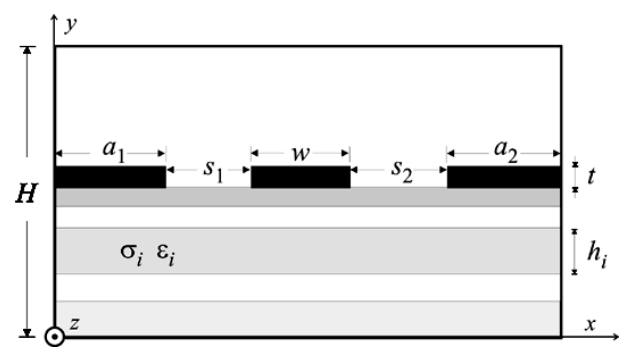

Fig. 1. Cross-sectional view of a $\mathrm{CPW}$ on a multilayered semiconductor substrate.

the unknown current density). After solving the integral equation, the longitudinal current densities both inside the nonperfect conductors and lossy substrate layers are obtained simultaneously. Despite the fact that the quasi-TM approach overcomes the lacks of the conventional quasi-TEM analysis to analyze semiconductor layers and nonperfect conductors, it still keeps the advantage of dealing with situations where imposed sources are present. (Specifically, the imposed source in the integral equation for the longitudinal current density will be expressed in terms of the quasi-static electric potential, previously computed as a first step in the analysis.) In this sense, the rather involved eigenvalue problem typical of the full-wave propagating system will not appear in this approach, and the numerical difficulties associated with the root-searching process in the complex plane are avoided. The method also has the additional advantage of providing not only the phase constant, but also the characteristic impedance as a direct result from the analysis. As will be shown in the results, the method provides accurate results for the three well-known operation zones in MIS lines (dielectric, slow-wave, and skin effect modes), and additionally properly accounts for the conductor losses.

\section{QUASI-TM APPROACH}

The cross-sectional view of the transmission line under study is shown in Fig. 1. Both the layers of the substrate and the metallic conductors can present ohmic losses. The magnetic permeability is assumed to be $\mu_{0}$ in all the structures. Although the generic line in Fig. 1 is a CPW, it also includes the single microstrip line as a particular case for $a_{1}=a_{2}=0$. The rectangular ground box enclosing the structure is assumed to be a perfect conductor. Laterally open lines can be also analyzed using high enough relative values for $a_{i}$ with respect to $s_{i}$ and $w$. Similarly, the effect of the top ground plane can be minimized, if required, by taking $H$ high enough in relation to the other dimensions in the structure (for usual dimensions, five times the substrate height suffices). Finally, a nonperfect ground plane can be simulated as an additional lossy layer with dielectric permittivity $\epsilon_{0}$. In the analysis of this generic line, an implicit field dependence of the type $\exp (-j \gamma z+j \omega t)$ will be assumed, where $\gamma$ is the propagation constant and $\omega$ is the angular frequency.

As in the quasi-TEM approach, it will be assumed that the operating frequencies are such that $\lambda \gg d$, where $d$ is the distance between the center conductor and the ground plane and $\lambda$ is the corresponding wavelength. At these relative low frequencies, the average value of the longitudinal fields (i.e., in the propagation direction $z$ axis in this paper) are much smaller than their transverse (normal to $z$ ) components. Consequently, the term $-j \omega \mu_{0} H_{z}$ can be neglected in the computation of the transverse electric field $\mathbf{E}_{t}$. On the contrary, although the longitudinal electric field in the substrate is also smaller than the average transverse electric field, the term $\sigma E_{z}$ ( $\sigma$ is the electric conductivity) can be relevant because of the possible high values of the electric conductivity of the semiconductor layers. Thus, the longitudinal electric current in the substrate must be included together with the longitudinal electric current inside the nonperfect metallic conductors to accurately compute the transverse magnetic field $\mathbf{H}_{t}$, as well as the longitudinal ohmic losses. In summary, the above approximations can be seen as a quasi-TM approach for the fundamental mode in the sense that the effect of $H_{z}$ is neglected in the range of frequencies under study, but the longitudinal electric field is taken into account in order to accurately compute the transverse magnetic field, as well as the longitudinal ohmic losses. The equations corresponding to the described quasi-TM approach will be presented in Section II-A. As it will be shown, these equations allow to compute the quasi-static electric potential and then, as a second step, the longitudinal electric current density in the line, with the previously computed quasi-static electric potential acting as an imposed source.

\section{A. Quasi-TM Equations}

As mentioned above, under the quasi-TM approach, the term $-j \omega \mu_{0} H_{z}$ can be neglected in the computation of transverse electric field for the fundamental mode. Moreover, practical conductors are actually good conductors (typically $\sigma \sim 10^{7} \mathrm{~S} / \mathrm{m}$ ) and the transverse electric field is much smaller than the longitudinal electric field inside of them [19]. Thus, the transverse electric field can be neglected inside the metallic conductors or, equivalently, the conductors can be seen as perfect ones when computing the transverse electric field in the line. Therefore, conventional quasi-TEM approach remains valid for computing the transverse electric field in the whole line, and the following equation applies:

$$
\nabla_{t} \times \mathbf{E}_{t}=0
$$

From this equation, it can be written $\mathbf{E}_{t}=-\nabla_{t} \phi$, where $\phi$ is the common quasi-static potential. Inside each homogenous layer, $\nabla_{t} \cdot \mathbf{E}_{t}=0$, which leads to

$$
\nabla_{t}^{2} \phi=0 .
$$

Equation (2) can be readily solved imposing $\phi$ as a constant in the center conductor $\phi=V_{c}$, zero in the remaining, and enforcing the usual boundary conditions of continuity of $\phi$ and continuity of the total (conduction and displacement) transverse current normal to the dielectric/dielectric interfaces.

Now, in order to improve the conventional quasi-TEM approach to compute $\mathbf{H}_{t}$ including the effect of the longitudinal currents, the following magnetostatic approximation is used:

$$
\nabla_{t} \times \mathbf{H}_{t}=\sigma \mathbf{E}_{z}
$$

which applies inside each layer, as well as inside the nonperfect conductors. In (3), it is assumed that only the effect of the lon- 
gitudinal ohmic currents is relevant and, thus, longitudinal displacement currents can be neglected everywhere in the structure.

Under the quasi-TM assumption $\nabla_{t} \cdot \mathbf{H}_{t}=0$ and, hence, the transverse magnetic field can be related to the vector potential as

$$
\hat{\mathbf{z}} \times \nabla_{t} A_{z}=-\mu_{0} \mathbf{H}_{t}
$$

where $\hat{\mathbf{z}}$ is the unit vector along the $z$-axis. Finally, from (3) and (4), the following equation is found for the $z$ component of the vector potential:

$$
\nabla_{t}^{2} A_{z}=-\mu_{0} \sigma E_{z}=-\mu_{0} J_{z} .
$$

Since the above equation involves two unknown functions $A_{z}$ and $E_{z}$, an additional equation is required. This additional equation comes from $E_{z}=-\partial A_{z} / \partial t-\partial \phi / \partial z$, which, for the assumed field dependence, is written as

$$
E_{z}=-j \omega A_{z}+j \gamma \phi
$$

Equations (2), (5), and (6) summarize the quasi-TM approach. Since the propagation constant appearing in (6) is indeed unknown, only normalized functions $A_{z} / \gamma, E_{z} / \gamma$, or $J_{z} / \gamma$ can be obtained from (5) and (6). Nevertheless, as shown in Section II-B, these normalized functions will suffice to compute the propagation constant and the characteristic impedance in the line.

At the light of the above two equations, there are three possibilities of analysis.

1) Solving for $A_{z} / \gamma$.

The equation for $A_{z} / \gamma$ is obtained by substituting (6) into (5), which leads to

$$
\nabla_{t}^{2}\left(A_{z} / \gamma\right)-j \omega \mu_{0} \sigma\left(A_{z} / \gamma\right)=-j \mu_{0} \sigma \phi
$$

This equation, together with the boundary conditions of continuity of $A_{z}$ and its derivative normal to the interfaces (dielectric/dielectric and dielectric/conductor), is an inhomogeneous diffusion equation where the term $j \mu_{0} \sigma \phi$ plays the role of an imposed source term. In the particular case of perfect conductors, the boundary conditions must be modified imposing the continuity of $A_{z}$ at the interfaces, the continuity of its derivative normal to the dielectric/dielectric interfaces, and fixing $A_{z} / \gamma$ as a constant in the perfect conductors (inside a perfect conductor $E_{z}=0$, and then from (6) $\left.A_{z} / \gamma=\phi / \omega\right)$.

2) Solving for $E_{z} / \gamma$.

If the expression for $A_{z}$ in terms of $E_{z}$ and $\phi$ from (6) is introduced into (7) taking into account that $\nabla_{t}^{2} \phi=0$, the following equation is reached:

$$
\nabla_{t}^{2} E_{z}-j \omega \mu_{0} \sigma E_{z}=0
$$

This diffusion equation should be solved imposing the continuity of $E_{z}$ at the interfaces (dielectric/dielectric and dielectric/conductor) and the discontinuity of the derivative of $E_{z}$ normal to these interfaces. Specifically, because of the continuity of the derivative of $A_{z}$ at the interfaces, from (6), it is easily obtained that

$$
\Delta\left[\frac{\partial E_{z}}{\partial n}\right]=\Delta\left[j \gamma \frac{\partial \phi}{\partial n}\right]
$$

where $n$ is the normal coordinate to the interface and $\Delta$ indicates the discontinuity at the considered interface. From (9), it can be concluded that, under the quasi-TM assumption, the discontinuity of the derivative of $E_{z}$ is caused by the discontinuity of the derivative of $j \gamma \phi$ normal to the interfaces or, equivalently, by the derivative along the $z$-direction (factor $j \gamma$ ) of the total charge, free charge, and polarization charge at the interfaces (term $\Delta[\partial \phi / \partial n])$. Actually, this term plays the role of imposed sources in the computation of $E_{z}$. In the particular case of perfect conductors, the computation of $E_{z} / \gamma$ would come from (9), the continuity of $E_{z}$ at the interfaces, and the condition $E_{z}=0$ on the perfect conductors.

3) Solving for $J_{z} / \gamma$.

Although the solution of (7) or (8) are two valid possibilities, when nonperfect metallic conductors are present, the values of $A_{z}$ or $E_{z}$ cannot be fixed as a constant on them, which is expected to make the analysis more involved. In this sense, the above two possibilities appear to be suitable for the perfect conductors case (the computation of $E_{z}$ in the perfect conductors case following the technique introduced in this paper is presented in Appendix A). On the contrary, for the nonperfect conductors case, the computation of $J_{z} / \gamma$ is found to be a more convenient choice. This third option leads to an integral equation for $J_{z} / \gamma$ that can be solved in a rather simple way. This integral equation is readily obtained from (6)

$\frac{J_{z}(\mathbf{r}) / \gamma}{\sigma(\mathbf{r})}=j \omega \mu_{0} \int_{S} G\left(\mathbf{r}, \mathbf{r}^{\prime}\right) J_{z}\left(\mathbf{r}^{\prime}\right) / \gamma \mathrm{d} S+j \phi(\mathbf{r})$

where $G\left(\mathbf{r}, \mathbf{r}^{\prime}\right)$ is the Green's function corresponding to (5) with the boundary condition $G\left(\mathbf{r}, \mathbf{r}^{\prime}\right)=0$ for $\mathbf{r}$ on the rectangular ground box enclosing the structure. It is interesting to notice that if transmission lines with perfect conductors were analyzed using this third option, the integral equation (10) would require to perform the integral including the surface currents on the perfect conductor, as well as the volume currents in the conductive layers, which would clearly increase the complexity of the analysis. For example, if a MoM was used, two different types of domain would be required: one for volumetric currents and other for surface currents. Thus, options 1) and 2) are definitely more convenient when dealing with perfect conductors. In this way, the authors have followed option 3) to analyze lines including lossy conductors, whereas the diffusion equation (8) for $E_{z}$ is solved to analyze lines with perfect conductors.

\section{B. Transmission-Line Model}

A transmission-line model based on the quasi-TM approach previously reported is presented here. The frequency-dependent 
parameters of this model will be obtained in terms of the electrostatic potential $\phi$ and the normalized function $J_{z} / \gamma$. The characteristic impedance, as well as the propagation constant in the line are then obtained in terms of the transmission-line parameters.

Applying Faraday's law to the line under study, it is found that

$$
\nabla_{t} \times \mathbf{E}_{z}-j \gamma \hat{\mathbf{z}} \times \mathbf{E}_{t}=-j \omega \mathbf{H}_{t}
$$

Performing a left cross product by vector $\hat{\mathbf{z}}$ and then a right cross product by $\mathbf{H}_{t}^{*}$, after integrating over the cross section of the line and after some algebra, (11) now becomes

$$
j \gamma P=4 j \omega\left[\left\langle W_{M}\right\rangle_{t}-\left\langle W_{E}\right\rangle_{l}\right]+2\left\langle P_{J}\right\rangle_{l}
$$

where $\left\langle W_{M}\right\rangle$ and $\left\langle W_{E}\right\rangle$ denote the time-average magnetic and electric energy p.u.l., respectively, $\left\langle P_{J}\right\rangle$ is the time-average Joule's power delivered p.u.l., subscripts $t$ and $l$ indicate that the corresponding magnitude is associated to the transversal or longitudinal fields, respectively, and $P$ is the usual complex power given by

$$
P=\int_{S}\left(\mathbf{E}_{t} \times \mathbf{H}_{t}^{*}\right) \cdot \mathrm{d} \mathbf{S}
$$

where $S$ indicates the cross section of the line. It is important to mention that the boundary condition $E_{z}=0$ on the rectangular box enclosing the line has been enforced to obtain (12), therefore, losses in the rectangular box have not been included in the analysis. Similarly, starting from Ampère-Maxwell's equation,

$$
\nabla_{t} \times \mathbf{H}_{z}-j \gamma \hat{\mathbf{z}} \times \mathbf{H}_{t}=(\sigma+j \omega \epsilon) \mathbf{E}_{t}
$$

and after operating in a similar way as above, the following equation is obtained:

$$
-j \gamma^{*} P=4 j \omega\left[\left\langle W_{M}\right\rangle_{l}-\left\langle W_{E}\right\rangle_{t}\right]+2\left\langle P_{J}\right\rangle_{t} .
$$

It is interesting to notice that the equation resulting from adding (12) to (15) could have been obtained from Poynting's theorem, but (12) and (15) cannot be obtained separately from this theorem. Under the current quasi-TM assumption, (12) and (15) reduce to

$$
\begin{gathered}
j \gamma P=4 j \omega\left\langle W_{M}\right\rangle_{t}+2\left\langle P_{J}\right\rangle_{l} \\
-j \gamma^{*} P=-4 j \omega\left\langle W_{E}\right\rangle_{t}+2\left\langle P_{J}\right\rangle_{t} .
\end{gathered}
$$

Note that the term $\left\langle W_{E}\right\rangle_{l}$, which would appear in (16) under the quasi-TM approximation, has been omitted because the longitudinal displacement currents are negligible assuming $\sigma \gg \omega \epsilon$, as mentioned in Section II-A. Starting from (16) and (17), the remainder of this section is devoted to develop a "consistent" transmission-line model. Here, the term "consistent" will mean that the voltage $V$ and the current $I$ in the line, as well as the frequency-dependent parameters (complex capacitance and inductance p.u.l.), will be defined in such a way that the conventional telegrapher's equations together with the additional equation

$$
P=V I^{*}
$$

are satisfied.
As mentioned in Section II-A, the transverse electric field is computed in the current quasi-TM approach in the same way as in the quasi-TEM approach or, equivalently, longitudinal currents are assumed negligible for the computation of $\mathbf{E}_{t}$. Therefore, the quasi-TEM definitions of both the voltage in the line and the complex shunt capacitance p.u.l. are found to be the most convenient choice. The voltage will then be defined as the quasi-static electric potential in the center conductor $V=V_{c}$, which is a constant over this conductor even for nonperfect conductors (see Section II-A). The corresponding expression for the complex shunt capacitance p.u.l. $\hat{C}=C-j G / \omega$ is given by

$$
C=\frac{1}{V} \int_{c} \epsilon \mathbf{E}_{t} \cdot \hat{\mathbf{n}} \mathrm{d} l \quad G=\frac{1}{V} \int_{c} \sigma \mathbf{E}_{t} \cdot \hat{\mathbf{n}} \mathrm{d} l
$$

where $c$ denotes the contour of the center conductor and $\hat{\mathbf{n}}$ is the outward unit vector normal to the center conductor. Next, the telegrapher's equation related to (17) can be easily obtained. Starting from the identity

$$
\int_{S}\left(\epsilon-j \frac{\sigma}{\omega}\right) \mathbf{E}_{t} \cdot \mathbf{E}_{t}^{*} \mathrm{~d} S=4\left\langle W_{E}\right\rangle_{t}-2 \frac{j}{\omega}\left\langle P_{J}\right\rangle_{t}
$$

and expressing $\mathbf{E}_{t}^{*}=-\nabla_{t} \phi^{*}$, using the identity $\mathbf{E}_{t} \cdot \nabla_{t} \phi^{*}=$ $\nabla_{t} \cdot\left(\phi^{*} \mathbf{E}_{t}\right)-\phi^{*} \nabla_{t} \cdot \mathbf{E}_{t}$ and $\nabla_{t} \cdot \mathbf{E}_{t}=0$, the integral in (20) can be written as

$$
\int_{S}\left(\epsilon-j \frac{\sigma}{\omega}\right) \mathbf{E}_{t} \cdot \mathbf{E}_{t}^{*} \mathrm{~d} S=-\int_{S}\left(\epsilon-j \frac{\sigma}{\omega}\right) \nabla_{t} \cdot\left(\phi^{*} \mathbf{E}_{t}\right) \mathrm{d} S .
$$

Expressing the resulting integral as a contour integral by means of the divergence theorem and substituting $\phi^{*}=V^{*}$ on the center conductor and $\phi^{*}=0$ on the grounded conductors, (20) can now be rewritten as

$$
V^{*} \int_{c}\left(\epsilon-j \frac{\sigma}{\omega}\right) \mathbf{E}_{t} \cdot \hat{\mathbf{n}} \mathrm{d} l=4\left\langle W_{E}\right\rangle_{t}-2 \frac{j}{\omega}\left\langle P_{J}\right\rangle_{t} .
$$

Substituting (19) for the complex capacitance into (22), the following expression is obtained:

$$
j \omega V^{*} V \hat{C}=4 j \omega\left\langle W_{E}\right\rangle_{t}+2\left\langle P_{J}\right\rangle_{t} .
$$

Finally, substituting (17) after complex conjugation in (23) and using (18), the telegrapher's equation is obtained as follows:

$$
j \gamma I=j \omega \hat{C} V .
$$

The definition of the current $I$ arises now as a consequence of the previous definition of $V$. Starting from (13) and (18), and after some mathematical manipulations (see Appendix B), the following expression is obtained:

$$
P=V I^{*}=V I_{c}^{*}+\int_{S(s)} J_{z}^{*} \phi \mathrm{d} S
$$

where $S(s)$ is the cross section corresponding to the conductive layers and $I_{c}$ is the current associated to the center conductor given by

$$
I_{c}=\oint_{c} \mathbf{H} \cdot \mathrm{d} \mathbf{l}=\int_{S(c)} J_{z} \mathrm{~d} S
$$

where $c$ is again the contour of the center conductor and $S(c)$ is its corresponding cross section. (In the particular case of perfect 
conductors $J_{z}=0$ inside them, $I_{c}$ can only be computed as a line integral around the contour of the center conductor.) The expression for the total current $I$ is now obtained from (25) after complex conjugation as

$$
I=I_{c}+\frac{1}{V^{*}} \int_{S(s)} J_{z} \phi^{*} \mathrm{~d} S .
$$

It should be observed that the above expression for the current includes a term (expressed as a surface integral) that does not appear under the conventional quasi-TEM approximation. From (27), it is interesting to emphasize that, under the current approach, the total current is not simply computed as the addition of the strip current to the substrate current resulting from performing the surface integral of $J_{z}$ over the cross section of the conductive layers. In the most general case of nonperfect metallic conductors where volumetric currents exist inside them, taking into account that $\phi=V$ on the center conductor, (27) can be written in a more compact way as

$$
I=\frac{1}{V^{*}} \int_{S^{\prime}} J_{z} \phi^{*} \mathrm{~d} S
$$

where $S^{\prime}$ includes the cross section of the center conductor and conductive layers. Expression (28) shows that, under the quasi-TM approach, $I$ can be seen as caused by an effective current density $J_{z \text {,eff }}$ expressed as the actual current density $J_{z}$ weighted by the factor $(\phi / V)^{*}$ as follows:

$$
J_{z, \mathrm{eff}}=J_{z}\left[\frac{\phi}{V}\right]^{*} \quad I=\int_{S^{\prime}} J_{z, \mathrm{eff}} \mathrm{d} S .
$$

Once the voltage and current have been defined, the proper expression for the complex series inductance p.u.l. $\hat{L}=L-$ $j R / \omega$ can be obtained enforcing the remaining telegrapher's equation

$$
j \gamma V=j \omega \hat{L} I
$$

and then

$$
\hat{L}=\frac{V}{\omega I / \gamma}
$$

As explained in Section II-A, it can be observed that it is only necessary to compute the normalized current $I / \gamma$ to obtain the complex series inductance p.u.l. in the line. Finally, it is interesting to mention that substituting (18) into (16) and (17), and using the telegrapher's equations (30) and (24) to eliminate $V$ and $I$ in (16) and (17), respectively, the following set of equations are obtained:

$$
\begin{aligned}
\left\langle W_{M}\right\rangle_{t} & =\frac{1}{4} L|I|^{2} & \left\langle W_{E}\right\rangle_{t} & =\frac{1}{4} C|V|^{2} \\
\left\langle P_{J}\right\rangle_{l} & =\frac{1}{2} R|I|^{2} & \left\langle P_{J}\right\rangle_{t} & =\frac{1}{2} G|V|^{2} .
\end{aligned}
$$

Therefore, it can be concluded that the previous definitions for $V$ and $I$ preserve the physical meaning of the transmission-line parameters. The propagation constant can now finally be obtained from the telegrapher's equations (24) and (30) as $\gamma=$ $\beta-j \alpha=\omega \sqrt{\hat{C} \hat{L}}$. Due to previous definitions, the characteristic impedance $Z_{0}=V / I=\sqrt{\hat{L} / \hat{C}}$ also has its usual meaning in terms of the complex power

$$
P=V I^{*}=Z_{0}|I|^{2}=\frac{|V|^{2}}{Z_{0}^{*}}
$$

\section{MoL/MoM Analysis}

Here, the integral equation (10) is solved by a mixed approach that combines the MoL with the MoM. In a first step, it is necessary to compute the potential $\phi$ as well as the Green's function appearing in (10). The first task was carried out in this study by solving (2) using the conventional MoL with equidistant discretization [20]. After applying this method, the potential $\phi$ is available along the vertical lines in all the structures. The computation of the Green's function was also carried out by the MoL. Since the explicit obtaining of Green's functions is beyond the usual scope of the MoL, the technique developed in this study will be explained in some detail. Thus, starting from (5) and following the usual techniques of the MoL, the second derivative operator $\partial^{2} / \partial x^{2}$ is discretized with the Dirichlet condition for the left and right walls ( $A_{z}=0$ on the walls). A system of $N$ coupled differential equations is then obtained, $N$ being the total number of lines. This system relates the components of the $x$-discretized function $A_{z}$, i.e., the $N$-elements column array $[A]$, to the $x$-discretized function $J_{z}, N$-elements column array $[J]$ (note that subscript $z$ has been omitted in the arrays resulting from discretization). As usual in the MoL, the $i$ th element of arrays $[A]$ and $[J], A_{i}(y)$ and $J_{i}(y)$, respectively, are the values of the corresponding magnitude along the $i$ th line. This system of coupled equations is then diagonalized with the proper eigenvector matrix $[T]$ where

$$
T_{i, j}=\sqrt{\frac{2}{N+1}} \sin \left(\frac{i j \pi}{N+1}\right)
$$

which leads to the following set of uncoupled equations:

$$
\frac{\partial^{2}}{\partial y^{2}} \tilde{A}_{i}(y)-k_{i}^{2} \tilde{A}_{i}(y)=-\mu_{0} \tilde{J}_{i}(y), \quad i=1, \ldots, N
$$

where ${ }^{\sim}$ indicates transformed magnitude $([\tilde{A}]=[T][A]$ and $[\tilde{J}]=[T][J])$, subscript $i$ refers to the $i$ th element of the transformed $N$-elements column array, and $k_{i}^{2}$ are the eigenvalues of the discretized operator, which for the actual boundary conditions are found to be

$$
k_{i}=\frac{2}{h} \sin \left(\frac{i \pi}{2(N+1)}\right)
$$

with $h$ being the discretization distance. The orthogonality of the symmetric eigenvector matrix $[T]=[T]^{t}=[T]^{-1}$ can be easily proven. This property will be used later in the analysis. The transformed Green's function associated to the $i$ th element $\tilde{G}_{i}\left(y, y^{\prime}\right)$ in (36) can be now obtained by solving

$$
\frac{\partial^{2}}{\partial y^{2}} \tilde{G}_{i}\left(y, y^{\prime}\right)-k_{i}^{2} \tilde{G}_{i}\left(y, y^{\prime}\right)=\delta\left(y-y^{\prime}\right)
$$


with the boundary condition $\tilde{G}_{i}\left(y, y^{\prime}\right)=0$ for $y=0$ and $y=$ $H$. Solving the above equation, the following expression has been obtained:

$$
\tilde{G}_{i}\left(y, y^{\prime}\right)= \begin{cases}\sinh \left[k_{i}\left(y^{\prime}-H\right)\right] \sinh \left(k_{i} y\right) / \eta_{i}, & y<y^{\prime} \\ \sinh \left[k_{i}(y-H)\right] \sinh \left(k_{i} y^{\prime}\right) / \eta_{i}, & y^{\prime}>y\end{cases}
$$

where $\eta_{i}=k_{i} \sinh \left(k_{i} H\right)$.

The MoM will next be applied to (10). First, the unknown transformed function $\tilde{J}_{i}(y)$ is expressed in terms of pulse basis functions as follows:

$$
\tilde{J}_{i}(y)=\sum_{m=1}^{M} \tilde{J}_{i, m} \tau\left(y_{m}\right)
$$

where pulse function $\tau\left(y_{m}\right)=1$ in the interval $y_{m}-a_{m} / 2<$ $y<y_{m}+a_{m} / 2$ and zero otherwise ( $y_{m}$ is the center of the domain and $a_{m}$ is its width). The unknown weights $\tilde{J}_{i, m}$ are constant inside each domain and $M$ is the total number of vertical domains. Next, point matching will be enforced in the centers of the vertical domains. In the center $\left(y_{n}\right)$ of the $n$th domain corresponding to the $j$ th component, the value of the function $\tilde{A}_{j}\left(y_{n}\right)$, denoted by $\tilde{A}_{j, n}$ from now on, can be expressed as

$$
\tilde{A}_{j, n}=-\mu_{0} \int_{0}^{H} \tilde{G}_{j}\left(y_{n}, y^{\prime}\right) \tilde{J}_{j}\left(y^{\prime}\right) \mathrm{d} y^{\prime} .
$$

Using the expansion (40) into basis functions, the following expression is obtained:

$$
\tilde{A}_{j, n}=-\mu_{0} \sum_{m=1}^{M} \tilde{g}_{j, n}^{m} \tilde{J}_{j, m}
$$

where

$$
\tilde{g}_{j, n}^{m}=\int_{y_{m}-a_{m} / 2}^{y_{m}+a_{m} / 2} \tilde{G}_{j}\left(y_{n}, y^{\prime}\right) \mathrm{d} y^{\prime} .
$$

The above integrals can be obtained in closed form, giving the following result:

$$
\begin{aligned}
\tilde{g}_{j, n}^{m}=\frac{-2 \sinh \left(k_{j}, d_{T}\right) \sinh \left(k_{j}, d_{B}\right)}{\eta_{j}} & \sinh \left(k_{j} \frac{a_{m}}{2}\right) \\
& +\frac{\delta_{n, m}}{k_{j}^{2}}\left[\cosh \left(\frac{k_{j} a_{m}}{2}\right)-1\right]
\end{aligned}
$$

where $\delta_{n, m}$ is the Kronecker delta, $d_{T}=H-y_{\max }$ and $d_{B}=$ $y_{\min }$ with $y_{\max }$ and $y_{\min }$ being the maximum and minimum between $y_{n}$ and $y_{m}$, respectively. Returning now to the spatial domain via the transformation matrix $[T]$, and after some algebra, (42) becomes

$$
A_{i, n}=-\mu_{0} \sum_{l=1}^{N} \sum_{m=1}^{M} g_{i, n}^{l, m} J_{l, m}
$$

where $A_{i, n}=A_{i}\left(y_{n}\right), J_{l, m}=J_{l}\left(y_{m}\right)$ and

$$
g_{i, n}^{l, m}=\sum_{j=1}^{N} T_{i, j} \tilde{g}_{j, n}^{m} T_{j, l} .
$$

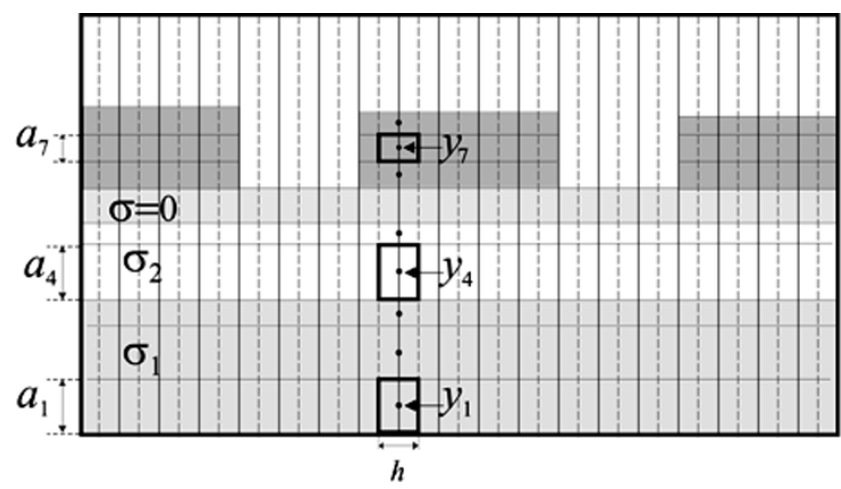

Fig. 2. Discretization pattern to apply the MoL/MoM technique. Two domains ( 1 and 4$)$ along a line, each of them in different conductive layers, have been remarked together with a domain inside the nonperfect center conductor 7. All the centers of the domains where $J_{z} \neq 0$ along the line have been also dotted.

Note that the orthogonality and symmetry of matrix $[T]$ have been used in this equation. Finally, substituting (45) into the integral equation (10), the following equation system is obtained:

$$
\frac{J_{i, n} / \gamma}{\sigma_{i, n}}=j \omega \mu_{0} \sum_{l=1}^{N} \sum_{m=1}^{M} g_{i, n}^{l, m} J_{l, m} / \gamma+j \phi_{i, n} .
$$

In the above expression, subscripts $i, n$ (or $l, m$ ) indicate that the corresponding magnitude is evaluated for $y=y_{n}$ in the $i$ th line (or $y=y_{m}$ in the $l$ th line). In Fig. 2, the discretization lines (vertical solid lines) used to apply the MoL has been drawn together with the centers of the domains along a line, and some of the domains on this line have also been remarked. After solving the equation's system (47), the normalized values $J_{i, n} / \gamma$ are finally obtained. Under the MoL scheme the final computation of the normalized current using (28) now reduces to an addition. $I / \gamma$ can then be straightforwardly computed as

$$
I / \gamma=\frac{1}{V^{*}} \sum_{i, n}\left(a_{n} h\right) \phi_{i, n}^{*} J_{i, n} / \gamma
$$

where the addition includes the domains in the conductive layers and center conductor. Finally, and previously to accomplish the analysis of any particular line, it is interesting to mention some general ideas about the discretization sizes in Fig. 2 to compute the normalized current. In this sense, for lossy conductors, the number of lines over the center strip, as well as the number of vertical domains along each line in the strip, should be chosen with reference to the typical distance involved in the problem, i.e., the skin depth in the strip at the operating frequency $\delta$. Specifically, a minimum of $3 \times w / \delta$ lines over the strip width and $3 \times t / \delta$ vertical domains along each line in the strip should be used in order to accurately enough model the current density inside the center strip. With regard to the conductive layers, similar to the metallic strip, in those particular situations where $h_{i} \sim \delta_{i}$ ( $\delta_{i}$ is the skin depth in the conductive layer and $h_{i}$ is its height), a minimum number of $3 \times h_{i} / \delta_{i}$ vertical domains should be used. Nevertheless, in most cases, $h_{i} \ll \delta_{i}$, and the current density inside of the conductive layers is expected to exhibit a smooth behavior, and a few number of vertical domains in the conductive layer will provide sufficient accuracy. 


\section{NUMERICAL RESULTS}

Results for three different examples will be presented here. The first example will show the capability of the current quasi-TM approach to provide accurate results for the propagation constant of the fundamental mode in a MIS line in the three different operating zones (dielectric, slow-wave, and skin-effect modes). In this first example, the conductors are assumed to be zero-thickness perfect conductors in order to compare with previous accurate full-wave SDA results. In the second example, and with the purpose of pointing out the capability of the current method to accurately enough compute the conductor losses, a single lossy microstrip in vacuum is considered. Our computed results will be compared with other results previously reported in papers specifically devoted to conductor losses. In the third example, three MIS CPW lines with nonperfect conductors are analyzed and the results are compared with previous computed and measured data.

The line under analysis in the first example is a MIS open microstrip line (see the inset in Fig. 3). The propagation constant of the fundamental mode has been computed as a function of the loss tangent of the semiconductor layer at $1 \mathrm{GHz}$ (increasing the loss tangent is equivalent to using a more conductive semiconductor). As the loss tangent increases, the fundamental mode is expected to change from a dielectric mode to a slow-wave mode and, finally, to a skin-effect mode. The results computed in this study are compared with previous full-wave SDA results in [7]. The metallic strip is assumed to be a zero thickness perfect conductor, as required by the standard SDA. Since the strip conductor is perfect, the diffusion equation for $E_{z}$, i.e., (8), has been solved instead of the integral equation (10), as mentioned in Section II-A. A maximum of $\sim 50$ lines over the strip in the MoL discretization was enough to provide three significant digits in the results in all cases. A laterally open condition has been simulated by using $a_{1}=a_{2}=0, s_{1}=s_{2}=5 \mathrm{w}$, and $H=5\left(h_{1}+h_{2}\right)$ to minimize the effect of the top ground plane (see Fig. 1). The results obtained for the real part of the relative effective dielectric permittivity $\epsilon_{r \text {,eff }}^{\prime}$ and for the attenuation are plotted in Fig. 3. As can be observed in this figure, a total agreement with the full-wave results in [7] is achieved for the three types of modes (dielectric, slow-wave, and skin-effect modes). The results obtained by a conventional quasi-TEM analysis have been also computed and represented by dashed lines. Clearly, quasi-TEM results are accurate in the dielectric-mode region and in the transition zone to the slow-wave mode; these results are also accurate for $\epsilon_{r, \text { eff }}^{\prime}$ in the slow-wave mode region, and also for the attenuation before reaching the minimum value in this zone. Finally, quasi-TEM results become totally erroneous from the transition zone to the skin-effect mode upward. Additionally, and in order to have an idea of the magnitude of the skin effect in the semiconductor layer, an upper axis has been plotted showing the ratio $h_{1} / \delta_{1}$, where $\delta_{1}$ is the skin depth in the semiconductor layer. It can be observed that $h_{1} / \delta_{1} \simeq 1$ indicates the transition from the slow-wave zone to the skin effect region. From this example, it can be concluded that the quasi-TM approach overcomes the deficiencies of the conventional quasi-TEM analysis to provide the propagation characteristics of the slow-wave and skin-effect modes in MIS lines.

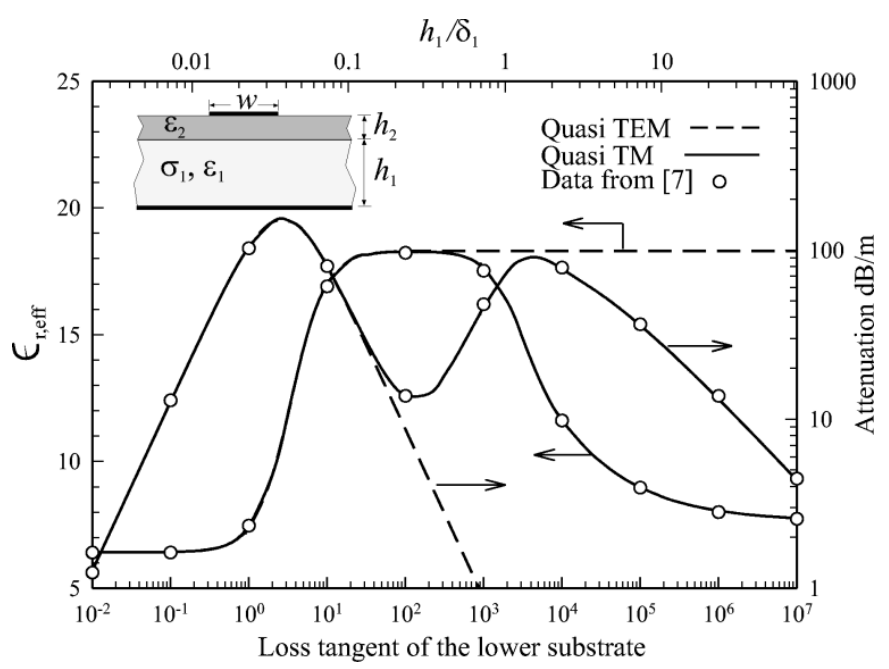

Fig. 3. Real part of the effective dielectric permittivity $\epsilon_{r, \text { eff }}^{\prime}$ and attenuation for the fundamental mode in an open MIS microstrip line as a function of the loss tangent of the lower layer at $1 \mathrm{GHz} . w=600 \mu \mathrm{m}, t=0 \mu \mathrm{m}, h_{1}=500 \mu \mathrm{m}$, $h_{2}=135 \mu \mathrm{m}$, and $\epsilon_{r 1}=\epsilon_{r 2}=9.7$.

As a second example, the resistance of an isolated microstrip in vacuum has been computed. This example has been previously analyzed in [21] by a variational method and in [22] by solving the diffusion equation (8) using a separation of variables technique and Green's function method. In this paper, the currents inside the strip have been obtained by solving integral equation (47). In this particular case where $J_{z}$ exists only inside the conductors, the potential $\phi$ in (47) is the potential in the strip $V_{c}$, which, under the current approximation, is assumed to be constant. The isolated microstrip has been simulated using the following relative values for the distances: $a_{1}=a_{2}=0, s_{1}=s_{2}=5 w, h_{1}=5 w$, and $H-\left(h_{1}+t\right)=5 w$ (see Fig. 1). As mentioned in Section III, the number of lines over the strip, as well as the number of vertical domains along each line in the strip have been chosen with reference to the typical distance involved in the problem, i.e., the skin depth in the strip at the operating frequency $\delta$. Specifically, $\sim 4 \times w / \delta$ lines over the strip width and $\sim 4 \times t / \delta$ vertical domains along each line in the strip sufficed to achieve three significant digits. In Fig. 4, the values for the ratio between the ac resistance and dc resistance p.u.l. $R_{\mathrm{ac}} / R_{\mathrm{dc}}$, obtained for different aspect ratio $w / t$, are plotted versus the normalized frequency $P=\sqrt{2 \mu_{0} \sigma w t f}$, where $f$ is the ordinary frequency. According to the principle of similitude (see [22] and the references therein) applied to an isolated microstrip, the normalized frequency has the advantage that strips with different values for $w, t$, and $\sigma$, but with the same aspect ratio, have the same value for $R_{\mathrm{ac}} / R_{\mathrm{dc}}$ at the same normalized frequency. Our results show a good agreement with results in [21] and [22] in the range of frequencies and aspect ratios considered in Fig. 4. In the case $w / t=1$ for $P<0.79$, the thickness is lesser than the skin depth $t / \delta<1$, and the line operates under a weak skin effect regime. On the contrary, for $P>4, t / \delta>5$, and a strong skin effect is present in the strip (it will be assumed $t / \delta>5$ as the condition for a strong skin effect). Thus, for $w / t=1$, the studied range of frequencies covers the weak skin-effect regime, strong skin-effect regime, and the transition between them. Under strong skin effect in the strip, the well-known Wheeler's incremental inductance rule can be used; specifically, the zone of constant slope in the case $w / t=1$ in 


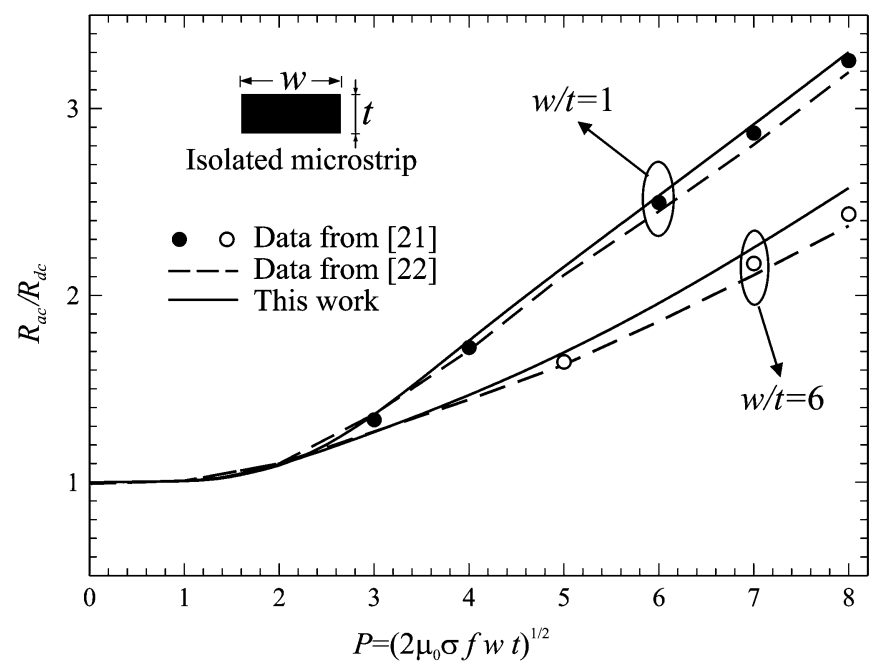

Fig. 4. $R_{\mathrm{ac}} / R_{\mathrm{dc}}$ for a single isolated microstrip in vacuum with different aspect ratio $w / t$ as a function of the normalized frequency $P=\sqrt{2 \mu_{0} \sigma w t f}$.

Fig. 4 indicates the zone of validity of the Wheeler's rule (under Wheeler's approach, $\left.R_{\mathrm{ac}} \propto f^{1 / 2}\right)$. Concerning the case of aspect ratio $w / t=6$ for $P<1.95, t / \delta<1$, and the line operates under a weak skin effect, and for $P>9.77, t / \delta>5$, which indicates a strong skin effect. Thus, in this case, the line does not operate under a strong skin-effect regime in the considered range of frequencies. It can be concluded that the current method accurately accounts for the conductor losses in all the possible skin-effect regimes. Finally, in microwave monolithic integrated circuits, the ratio $t / \delta$ ranges from $\sim 1$ at $1 \mathrm{GHz}$ to $\sim 5$ at $20 \mathrm{GHz}$, assuming that the metallization conductivity is $\sim 4 \times 10^{7} \mathrm{~S} / \mathrm{m}$ and $t \sim 3 \mu \mathrm{m}$. Consequently, Wheeler's incremental rule—generally a good approach to analyze thin-film circuits-is expected to provide accurate enough results in MMICs only for frequencies up to 20 $\mathrm{GHz}$. This is an important reason to dispose of efficient methods to compute the conductor losses under a weak skin effect.

As a third example, three MIS CPWs are analyzed. The selected values for the resistivity $\rho$ of the silicon layer were chosen in order to deal with very weakly, weakly, and heavily doped structures. In the three cases, the metallizations are printed on a thin layer of $\mathrm{SiO}_{2}$ above the doped silicon layer. To simulate the open structure, the relative dimensions are set to $a_{1}=a_{2}=$ $4(w+s), H-\left(h_{1}+h_{2}+t\right)=6 w$ (see Fig. 1). In all cases, the results obtained are compared with previous reported data.

In Fig. 5(a) and (b), the results for the slow-wave factor and attenuation corresponding to the weakly $\rho=1 \Omega \cdot \mathrm{cm}$, and very weakly doped $\rho=1000 \Omega \cdot \mathrm{cm}$ structures are plotted. As can be observed, the results in this study show a very good agreement with those computed in [10], except for small discrepancies in the slow-wave factor below $1 \mathrm{GHz}$ in the very weakly doped case. The results in [10] have been computed by means of a full-wave analysis by the MoL. The nature of the fundamental mode in the structures under study depends on the operating frequency. Thus, for frequencies higher than the dielectric relaxation frequency in the $\operatorname{Si}\left(f_{e}=1 /(2 \rho \pi \epsilon)\right)$, the line operates in the dielectric mode, whereas for frequencies lower than $f_{e}$, the line gets into the slow-wave zone. Specifically, the dielectric relaxation frequencies are $f_{e} \sim 150 \mathrm{MHz}$ for $\rho=1000 \Omega \cdot \mathrm{cm}$ and $\sim 150 \mathrm{GHz}$ for $1 \Omega \cdot \mathrm{cm}$. Therefore,

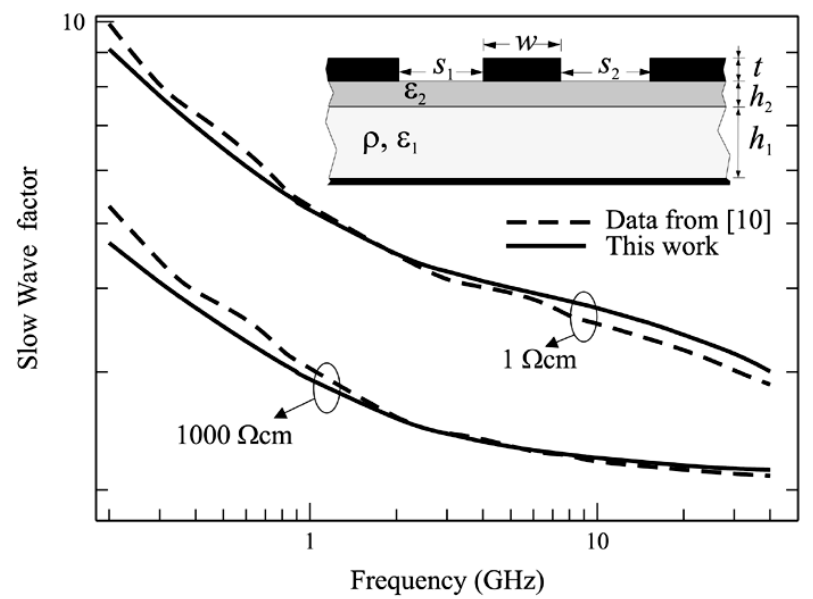

(a)

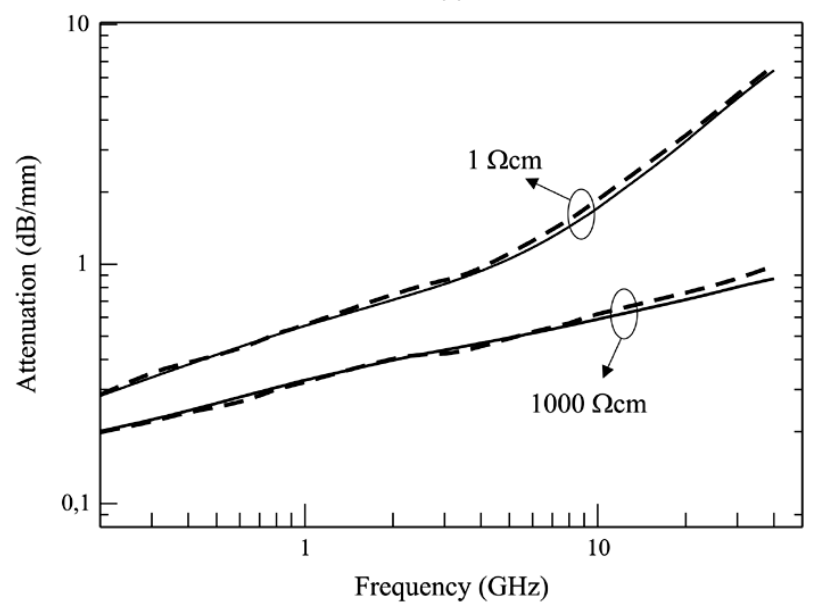

(b)

Fig. 5. (a) Slow-wave factor and (b) attenuation for the fundamental mode in a MIS CPW with lossy conductors versus the frequency for two different values of the resistivity $\rho$ of the semiconductor layer. Solid lines: this study. Dashed lines: data in [10]. Relative dielectric permittivities $\epsilon_{r 1}=12, \epsilon_{r 2}=4$ and metallic conductors conductivity $2.7 \times 10^{7} \mathrm{~S} / \mathrm{m}$ (low-density aluminum). Dimensions in micrometers: $w=10, t=0.8, s_{1}=s_{2}=5, h_{1}=480, h_{2}=1$.

in the very weakly doped structure, the fundamental mode is a dielectric one, whereas in the weakly doped structure, the fundamental mode is a slow-wave mode. To apply the MoL/MoM technique, the number of lines, as well as the number of vertical domains along each line was chosen to achieve convergence with three significant digits in the results. Specifically, 32 lines over the strip width at the lower frequency $(200 \mathrm{MHz})$ and 62 for the higher frequency $(40 \mathrm{GHz})$ were employed. The number of vertical domains used along each line over the metallic conductors was 2 at $200 \mathrm{MHz}$ and 5 at $40 \mathrm{GHz}$. Finally, three vertical domains along each line over the semiconductor layer sufficed to achieve the aforementioned accuracy for any value of the frequency considered. That means a total number of vertical domains on each line that ranges from 5 to 8 , depending on the operating frequency. The reason to use a greater number of lines and vertical domains at higher frequencies is to guarantee that a minimum of $\sim 3 \times w / \delta$ ( $\delta$ is the skin depth in the metallizations) lines over the strip width and a minimum of $\sim 3 \times t / \delta$ vertical domain along each line on the strip are used in order to solve the integral equation accurately inside the metallizations. In this sense, the authors have checked that 32 lines at $40 \mathrm{GHz}$ 


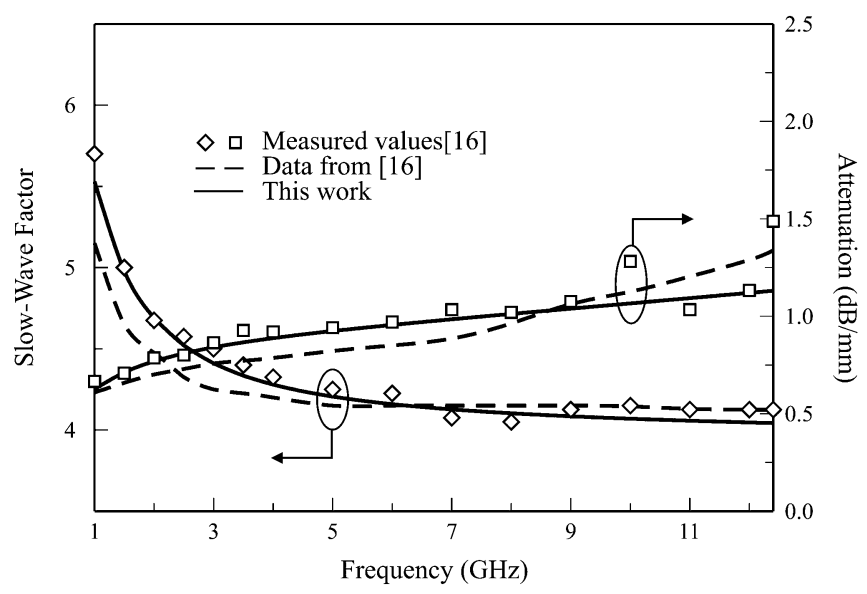

(a)

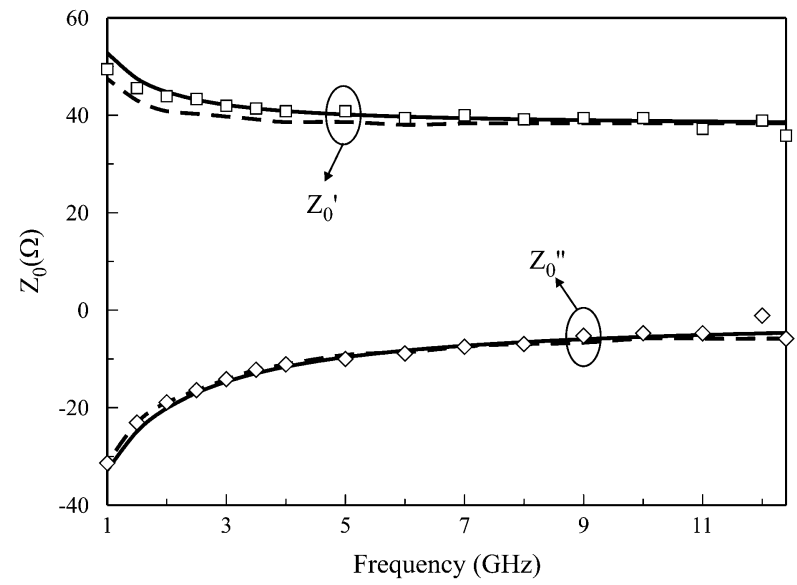

(b)

Fig. 6. (a) Slow-wave factor and attenuation and (b) characteristic impedance for the fundamental mode in a heavily doped MIS CPW with lossy conductors versus the frequency. Relative dielectric permittivities $\epsilon_{r 1}=12, \epsilon_{r 2}=4$ and metallic conductors conductivity $3.33 \times 10^{4} \mathrm{~S} / \mathrm{mm}$ (aluminum). Dimensions in micrometers: $w=4.2, t=1, s_{1}=s_{2}=6, h_{1}=530, h_{2}=0.53$. Resistivity of the semiconductor layer $\rho=0.0125 \Omega \cdot \mathrm{cm}$.

provides three digits in the results for the shunt capacitance and admittance p.u.l. and even for the series inductance p.u.l., but only two significant digits in the resistance.

To complete the current example, in Fig. 6(a) and (b), the results corresponding to a heavily doped silicon layer $\rho=0.0125 \Omega \cdot \mathrm{cm}$ in a MIS CPW are shown and compared with those computed and measured in [16]. In [16], the authors study the slow-wave propagation completing the usual quasi-TEM approach with a very simple correction to account for the losses associated to the longitudinal currents in the line. In this example, the dielectric relaxation frequency of the doped silicon is $\simeq 11980 \mathrm{GHz}$, then the fundamental mode is a slow-wave mode in the whole range of frequencies considered (from 1 to $12.4 \mathrm{GHz}$ ), as required by the model in [16]. In this structure, the results for the characteristic impedance $Z_{0}=Z_{0}^{\prime}+j Z_{0}^{\prime \prime}$ are also reported in [16] and have been plotted in Fig. 6(b). As seen in Fig. 6(a) and (b), the results in this study show a very good agreement with those in [16], specially with the measured ones.

From the above comparisons, together with the results in the previous examples, it can be concluded that the current model can be efficiently applied to the analysis of the three propagations modes (dielectric, slow-wave, and skin-effect modes) present in the most technologically interesting MIS structures with the already mentioned advantage of the direct computation of the characteristic impedance.

\section{CONCLUSION}

This paper has presented an efficient quasi-TM approach for the analysis of planar transmission lines on a layered substrate including semiconductor and nonperfect metallic conductors. A quasi-TM transmission-line model for the fundamental mode has been proposed by properly defining the voltage and current in the line, as well as the frequency-dependent transmission-line parameters. In order to compute the transmission-line parameters, the quasi-static electric potential and the longitudinal current density have been obtained by a hybrid MoL/MoM technique. Under the current quasi-TM approach, the equations to compute both the quasi-static electric potential and longitudinal current density are inhomogeneous. In this sense, the numerical difficulties appearing in the root-finding process inherent to a full-wave propagation analysis (eigenvalue equations) are avoided. Thus, the proposed method has the advantages of a conventional quasi-TEM analysis, whereas it overcomes its inability to accurately compute the series inductance and resistance p.u.l. when a semiconductor and/or nonperfect metallic conductors are present. The comparisons with previous results have shown the validity of the model to compute the propagation characteristics and characteristic impedance of the fundamental mode in MIS lines for the three possible cases, i.e., dielectric, slow-wave, and skin-effect modes. In conclusion, the method can be seen as a useful alternative to the more involved and time-consuming full-wave techniques with the additional advantage of the direct computation of characteristic impedance of the lines.

\section{APPENDIX A}

Here, we will explain the algorithm based on the MoL employed in this study to solve the diffusion equation (8) to compute $E_{z} / \gamma$ in a multilayered planar transmission line with perfect conductors. As will be made clear, the boundary condition of discontinuity of the derivative of $E_{z}$ makes the current algorithm different from other cases, and to some extent, also more involved. Specifically, due to the approach used in this study, the discontinuities of the derivative at the interfaces can be regarded as imposed superficial sources for $E_{z} / \gamma$. This is not commonly found in other cases where the continuity of the corresponding magnitude and its derivative at the interfaces of the substrate give rise to algorithms formally more compact and easy to implement in a computer code. Since thick perfect conductors do not change the way of accounting for the imposed sources in the substrate, the conductors will be assumed to be infinitesimally thin in order to simplify the explanation. Finally, it is interesting to remark that the quasi-static electric potential, as well as the complex displacement vector are assumed to be previously known to solve the diffusion equation (8).

Let us consider the diffusion equation (8) inside the $m$ th layer of the substrate of Fig. 1. In order to apply the MoL with $N$ equidistant lines, we define the $N$-elements column array $[E]$ 
whose $i$ th element $E_{i}(y)$ is the value of function $E_{z}$ along the $i$ th line (subscript $z$ will be omitted in the arrays resulting after $x$-discretization of $E_{z}$ and $A_{z}$ and its corresponding derivatives). After $x$-discretization of the $\nabla_{t}^{2}$ operator with Dirichlet conditions on the lateral walls, a system of $N$ coupled equations for $E_{i}(y)$ is then obtained and then diagonalized with the same eigenvector matrix $[T]$, as in (36). Thus, the following set of $N$ uncoupled equations are then obtained for the components of the transformed column array $[\tilde{E}]=[T][E]$ :

$$
\frac{\partial^{2}}{\partial y^{2}} \tilde{E}_{i}(y)-k_{i, m}^{2} \tilde{E}_{i}(y)=0, \quad i=1, \ldots, N
$$

with

$$
k_{i, m}=\sqrt{\frac{4}{h^{2}} \sin ^{2}\left(\frac{i \pi}{2(N+1)}\right)-j \omega \mu_{0} \sigma_{m}}
$$

where $\sigma_{m}$ is the conductivity of the $m$ th layer in the substrate. Solving (49), it is readily found that $\tilde{E}_{i}$ can be expressed as $\tilde{E}_{i}=a_{i} \sinh \left(k_{i, m} y\right)+b_{i} \cosh \left(k_{i, m} y\right)$. From this expression, the column array $[\tilde{E}]$ and its derivative $\left[\tilde{E}^{\prime}\right]=\partial[\tilde{E}] / \partial y$ (the symbol / will be used from now on to denote $\partial / \partial y$ ), at the top and bottom interfaces $(m)$ and $(m-1)$ (subscripts in parentheses will be used to denote interfaces) limiting the $m$ th layer can be related, after eliminating the coefficients $a_{i}$ and $b_{i}$, as

$$
[\tilde{\mathcal{E}}]_{(m)^{-}}=[\tilde{R}]_{m}[\tilde{\mathcal{E}}]_{(m-1)^{+}}
$$

where the $2 \times N$-elements column array $[\tilde{\mathcal{E}}]$ is given by

$$
[\tilde{\mathcal{E}}]=\left[\begin{array}{c}
{[\tilde{E}]} \\
{\left[\tilde{E}^{\prime}\right]}
\end{array}\right]
$$

subscripts - and + indicate the lower and upper faces of the interface, respectively, and $[\tilde{R}]_{m}$ is a four $N \times N$ diagonal boxes array with

$$
\begin{aligned}
& {[\tilde{R}]_{11, m}=\operatorname{diag}\left\{\cosh \left(k_{i, m} h_{m}\right)\right\}=[\tilde{R}]_{22, m}} \\
& {[\tilde{R}]_{12, m}=\operatorname{diag}\left\{\sinh \left(k_{i, m} h_{m}\right) / k_{i, m}\right\}} \\
& {[\tilde{R}]_{21, m}=\operatorname{diag}\left\{k_{i, m} \sinh \left(k_{i, m} h_{m}\right)\right\}}
\end{aligned}
$$

where $h_{m}$ is the height of the $m$ th layer.

Let us now consider that $(m)$ is an interface without conductors or, equivalently, $(m)$ is neither the lower ground plane (interface $(0)$, nor the upper interface of the substrate (interface $(M)$ ). Then

$$
\gamma[\tilde{\Delta}]_{(m)}=[\tilde{\mathcal{E}}]_{(m)^{+}}-[\tilde{\mathcal{E}}]_{(m)^{-}}
$$

is defined at interface $(m)$ to account for the discontinuity in the derivative $\left[\tilde{E}^{\prime}\right]$. Substituting $\left[\tilde{E}^{\prime}\right]=-j \omega\left[\tilde{A}^{\prime}\right]+j \gamma\left[\tilde{\phi}^{\prime}\right]$ in $[\tilde{\mathcal{E}}]$, assuming the continuity of $\left[\tilde{A}^{\prime}\right]$ at the interfaces without conductors, writing $\left[\tilde{\phi}^{\prime}\right]$ in terms of normal component to the interface of the complex displacement vector, and also assuming the continuity of this latter vector at this interface, the following expression is now obtained:

$$
[\tilde{\Delta}]_{(m)}=\left[\begin{array}{c}
{[0]} \\
-j[\tilde{D}]_{(m)} \zeta_{(m)}
\end{array}\right]
$$

where $[\tilde{D}]_{(m)}$ is the transformed of the normal component to interface $(m)$ of the complex displacement column array (note that subscript $y$ corresponding to normal direction has been omitted) and

$$
\zeta_{(m)}=\left(\epsilon_{m+1}-j \sigma_{m+1} / \omega\right)^{-1}-\left(\epsilon_{m}-j \sigma_{m} / \omega\right)^{-1} .
$$

Null column array [0] in (55) appears as a consequence of the continuity of $[\tilde{E}]$ at the interfaces. From the above equations, it can be finally written

$$
[\tilde{\mathcal{E}}]_{(m)^{+}}=[\tilde{R}]_{m}[\tilde{\mathcal{E}}]_{(m-1)^{+}}+\gamma[\tilde{\Delta}]_{(m)} .
$$

Starting from the upper face of the lower ground plane, interface $(0)^{+}$, and applying (57) until reaching the lower face of the upper interface of the substrate $(M)^{-}$, it is now obtained that

$$
[\tilde{\mathcal{E}}]_{(M)^{-}}=[\tilde{R}][\tilde{\mathcal{E}}]_{(0)+}+\gamma[\tilde{\chi}]
$$

where the total four $N \times N$ diagonal boxes array $[\tilde{R}]$ is given by

$$
[\tilde{R}]=\prod_{m=M}^{1}[\tilde{R}]_{m}
$$

with $2 \times N$ elements column array

$$
[\tilde{\chi}]=\sum_{k=1}^{M-1} \prod_{m=M}^{k+1}[\tilde{R}]_{m}[\tilde{\Delta}]_{(k)} .
$$

Substituting $[\tilde{E}]_{(0)^{+}}=[0]$ in $[\tilde{\mathcal{E}}]_{(0)^{+}}$for the current boundary condition (perfect ground plane), and operating in (58) to eliminate the vector $\left[\tilde{E}^{\prime}\right]_{(0)^{+}}$appearing in $[\tilde{\mathcal{E}}]_{(0)+}$, the following expression is finally obtained:

$$
\left[\tilde{E}^{\prime}\right]_{(M)^{-}}=[\tilde{U}][\tilde{E}]_{(M)}+\gamma[\tilde{\varphi}]
$$

where matrix $[\tilde{U}]=[\tilde{R}]_{22}[\tilde{R}]_{12}^{-1}$ and $[\tilde{\varphi}]=[\tilde{\chi}]_{2}-[\tilde{U}][\tilde{\chi}]_{1}$ with $[\tilde{\chi}]_{1}$ being an $N$-elements column array that contains the first $N$ elements of $[\tilde{\chi}]$, and $[\tilde{\chi}]_{2}$ being the remaining $N$ elements.

$\left[\tilde{E}^{\prime}\right]_{(M)}$ in the upper face of interface $(M)$ will now be related with $[\tilde{E}]_{(M)}$. Thus, regarding the space over the substrate as a vacuum layer $(M+1$ th) and applying the relation given by (51), it is easily obtained as follows:

$$
[\tilde{\mathcal{E}}]_{(M)^{+}}=[\tilde{S}][\tilde{\mathcal{E}}]_{(M+1)^{-}}
$$

where $[\tilde{S}]=[\tilde{R}]_{M+1}^{-1}$ is also a four $N \times N$ diagonal boxes array. For the current boundary condition at the top perfect ground plane, interface $(M+1),[\tilde{E}]_{(M+1)^{-}}=[0]$. This can be used to eliminate $\left[\tilde{E}^{\prime}\right]_{(M+1)^{-}}$in (62) to obtain

$$
\left[\tilde{E}^{\prime}\right]_{(M)^{+}}=[\tilde{Q}][\tilde{E}]_{(M)}
$$

where, in terms of the $N \times N$ diagonal boxes of matrix $[\tilde{S}],[\tilde{Q}]=[\tilde{S}]_{22}[\tilde{S}]_{12}^{-1}$.

Subtracting (61) from (63), transforming the resulting expression to the spatial domain by the eigenvector matrix $[T]$ and taking into account that $E_{z}=0$ on the conductors, the following expression is obtained:

$$
-j \gamma \zeta_{(M)}[D]_{\text {gap }}=[G]^{-1}[E]_{\text {gap }}-\gamma[\varphi]_{\text {gap }}
$$


where arrays with subscript "gap" contain only the values of the corresponding array on the gaps, $[G]^{-1}$ is the matrix resulting from omitting the elements of matrix $[Q]-[U]=[T]([\tilde{Q}]-$ $[\tilde{U}])[T]$ if at least one of its subscripts refers to a line on the conductors and column array $-j \gamma \zeta_{(M)}[D]_{\text {gap }}=\left[E_{(M)^{+}}^{\prime}-\right.$ $\left.E_{(M)^{-}}^{\prime}\right]_{\text {gap }}$, where $\zeta_{(M)}=\epsilon_{0}^{-1}-\left(\epsilon_{M}-j \sigma_{M} / \omega\right)^{-1}$ according to (56). Finally,

$$
[E]_{\text {gap }} / \gamma=[G]\left([\varphi]_{\text {gap }}-j \zeta_{(M)}[D]_{\text {gap }}\right) .
$$

From the normalized values for $E_{z}$ on the gaps at interface $(M),[E]_{\text {gap }} / \gamma$, and taking into account that the remaining elements of array $[E]_{(M)} / \gamma$ corresponding to the values of $E_{z}$ on the perfect metallic conductors are zero, $[E]_{(M)} / \gamma$ is finally obtained. Once $[E]_{(M)} / \gamma$ is available, the function $E_{i}(y) / \gamma$ along the $i$ th line can be computed, if necessary, at any value of $y$.

\section{APPENDIX B}

From (13) and substituting $\mathbf{E}_{t}=-\nabla_{t} \phi$, the following expression is obtained:

$$
P=\int_{S}\left(\mathbf{E}_{t} \times \mathbf{H}_{t}^{*}\right) \cdot \mathrm{d} \mathbf{S}=-\int_{S(s)}\left(\nabla_{t} \phi \times \mathbf{H}_{t}^{*}\right) \cdot \mathrm{d} \mathbf{S}
$$

where $S(s)$ denotes the cross-sectional part excluding the metallic conductors. The cross section of the metallic conductors can be excluded because $\mathbf{E}_{t}$ is assumed to be zero inside them under the current approach. Using the identity

$$
-\nabla_{t} \phi \times \mathbf{H}_{t}^{*}=-\nabla_{t} \times\left(\phi \mathbf{H}_{t}^{*}\right)+\phi \nabla_{t} \times \mathbf{H}_{t}^{*}
$$

integral, (66) can now be split into two terms as follows:

$$
P=-\int_{S(s)}\left(\nabla_{t} \times\left(\phi \mathbf{H}_{t}^{*}\right)\right) \cdot \mathrm{d} \mathbf{S}+\int_{S(s)}\left(\phi \nabla_{t} \times \mathbf{H}_{t}^{*}\right) \cdot \mathrm{d} \mathbf{S} .
$$

The first integral in the second member can be written as a contour integral

$$
-\int_{S(s)}\left(\nabla_{t} \times\left(\phi \mathbf{H}_{t}^{*}\right)\right) \cdot \mathrm{d} \mathbf{S}=\int_{c} \phi \mathbf{H}_{t}^{*} \cdot \mathrm{d} \mathbf{l}+\int_{g} \phi \mathbf{H}_{t}^{*} \cdot \mathrm{d} \mathbf{l}
$$

where subscript $c$ indicates contour of the center conductor and subscript $g$ contour of the grounded conductors. Notice that the vector $\mathrm{d} \mathbf{S}$ and $\mathrm{dl}$ are not related via the right-hand rule, but opposite, because of the minus sign in the surface integral. Taking into account that $\phi=0$ in the grounded conductors is a constant in the center conductor $\phi=V_{c}=V$, (69) becomes

$$
-\int_{S(s)}\left(\nabla_{t} \times\left(\phi \mathbf{H}_{t}^{*}\right)\right) \cdot \mathrm{d} \mathbf{S}=V I_{c}^{*}
$$

where the center conductor current $I_{c}$ has its habitual meaning

$$
I_{c}=\int_{c} \mathbf{H}_{t} \cdot \mathrm{d} \mathbf{l} \text {. }
$$

If the center conductor is a nonperfect one, $I_{c}$ can be written in terms of $J_{z}$ inside it as follows:

$$
I_{c}=\int_{c} \mathbf{H}_{t} \cdot \mathrm{d} \mathbf{l}=\int_{S(c)}\left(\nabla_{t} \times \mathbf{H}_{t}\right) \cdot \mathrm{d} \mathbf{S}=\int_{S(c)} J_{z} \mathrm{~d} S
$$

where $S(c)$ indicates the cross section of the center conductor. Substituting $P=V I^{*}$ and $\nabla_{t} \times \mathbf{H}_{t}=J_{z} \hat{\mathbf{z}}$ in (68), this equation now reads

$$
V I^{*}=V I_{c}^{*}+\int_{S(s)} J_{z}^{*} \phi \mathrm{d} S .
$$

Finally, from this equation, the expression for the current in the line $I$ is easily obtained as

$$
I=I_{c}+\frac{1}{V^{*}} \int_{S(s)} J_{z} \phi^{*} \mathrm{~d} S .
$$

\section{REFERENCES}

[1] H. Hasegawa and S. Seki, "Analysis of interconnection delay on very high-speed LSI/VLSI chips using an MIS microstrip line model," IEEE Trans. Microw. Theory Tech., vol. MTT-32, no. 12, pp. 1721-1727, Dec. 1984.

[2] H. Guckel, P. A. Brennan, and I. Palócz, "A parallel-plate waveguide approach to microminiaturized, planar transmission lines for integrated circuits," IEEE Trans. Microw. Theory Tech., vol. MTT-15, no. 8, pp. 468-476, Aug. 1967.

[3] H. Hasegawa, M. Furukawa, and H. Yanai, "Properties of microstrip lines on $\mathrm{Si}-\mathrm{SiO}_{2}$ system," IEEE Trans. Microw. Theory Tech., vol. MTT-19, no. 11, pp. 869-881, Nov. 1971.

[4] Y. Fukukoa, Y. Shih, and T. Itoh, "Analysis of slow-wave coplanar waveguides for monolithic integrated circuits," IEEE Trans. Microw. Theory Tech., vol. MTT-31, no. 7, pp. 567-573, Jul. 1983.

[5] R. Sorrentino, G. Leuzzi, and A. Silbermann, "Characteristics of metal-insulator-semiconductor waveguides for monolithic microwave circuits," IEEE Trans. Microw. Theory Tech., vol. MTT-32, no. 4, pp. 410-416, Apr. 1984.

[6] K. Wu and R. Vahldieck, "Propagation characteristics of MIS transmission lines with inhomogeneous doping profile," IEEE Trans. Microw. Theory Tech., vol. 38, no. 12, pp. 1872-1878, Dec. 1990.

[7] J. P. K. Gilb and C. A. Balanis, "MIS slow-wave structure over a wide range of parameters," IEEE Trans. Microw. Theory Tech., vol. 40, no. 12, pp. 2148-2154, Dec. 1992.

[8] F. L. Mesa, G. Cano, F. Medina, R. Marqués, and M. Horno, "On the quasi-TEM and full-wave approaches applied to coplanar multistrip on lossy dielectric media," IEEE Trans. Microw. Theory Tech., vol. 40, no. 3, pp. 524-531, Mar. 1992.

[9] F. Schmückle and R. Pregla, "The method of the lines for the analysis of lossy planar waveguides," IEEE Trans. Microw. Theory Tech., vol. 38, no. 10, pp. 1473-1479, Oct. 1990.

[10] K. Wu and R. Vahldieck, "Hybrid-mode analysis of homogeneously and inhomogeneouly doped low-loss slow-wave coplanar transmission lines," IEEE Trans. Microw. Theory Tech., vol. 39, no. 8, pp. 1348-1360, Aug. 1991.

[11] T. Shibata and E. Sano, "Characterization of MIS coplanar transmission lines for investigation of signal propagation in integrated circuits," IEEE Trans. Microw. Theory Tech., vol. 38, no. 7, pp. 881-890, Jul. 1990.

[12] J. F. Lee, "Finite element analysis of lossy dielectric waveguides," IEEE Trans. Microw. Theory Tech., vol. 42, no. 6, pp. 1025-1031, Jun. 1994.

[13] J. J. Kucera and R. J. Gutmann, "Effect of finite metallization and inhomogeneous dopings on slow-wave-mode propagation," IEEE Trans. Microw. Theory Tech., vol. 45, no. 10, pp. 1807-1810, Oct. 1997.

[14] E. Tuncer and D. P. Neikirk, "Highly accurate quasi-static modeling of microstrip lines over lossy substrates," IEEE Microw. Guided Wave Lett., vol. 2, no. 10, pp. 409-411, Oct. 1992.

[15] J. Aguilera, R. Marqués, and M. Horno, "Improved quasi-static spectral domain analysis of microstrip lines on high-conductivity insulator-semiconductor substrates," IEEE Microw. Guided Wave Lett., vol. 9, no. 2, pp. 57-59, Feb. 1999

[16] Y. R. Kwon, V. M. Hietala, and K. S. Champlin, "Quasi-TEM analysis of slow-wave mode propagation on coplanar microstructure MIS transmission lines," IEEE Trans. Microw. Theory Tech., vol. MTT-35, no. 6, pp. 545-551, Jun. 1987.

[17] J. Zheng, V. K. Tripathi, and A. Weisshaar, "Characterization and modeling of multiple coupled on-chip interconnects on silicon substrate," IEEE Trans. Microw. Theory Tech., vol. 49, no. 10, pp. 1733-1739, Oct. 2001. 
[18] F. Olyslager, Electromagnetic Waveguides and Transmission Lines. Oxford, U.K.: Oxford Univ. Press, 1999.

[19] J. D. Jackson, Classical Electrodynamics, 2nd ed. New York: Wiley, 1975.

[20] R. Pregla and W. Pascher, "The method of lines," in Numerical Techniques for Microwave and Millimeter Wave Passive Structures, T. Itoh, Ed. New York: J. Wiley, 1989, pp. 381-446.

[21] P. Waldow and I. Wolff, "The skin-effect at high frequencies," IEEE Trans. Microw. Theory Tech., vol. MTT-33, no. 10, pp. 1076-1082, Oct. 1985.

[22] R. Faraji-Dana and Y. L. Chow, "The current distribution and AC resistance of a microstrip structure," IEEE Trans. Microw. Theory Tech., vol. 38, no. 9, pp. 1268-1276, Sep. 1990.

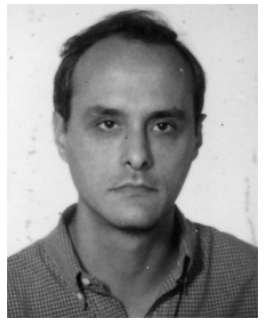

Gonzalo Plaza was born in Cádiz, Spain, in November 1960. He received the Licenciado and Doctor degrees in physics from the University of Seville, Seville, Spain in 1986 and 1995, respectively.

He is currently an Associate Professor with the Department of Applied Physics I, University of Seville. His research interest focuses on electromagnetic propagation in planar transmission lines.

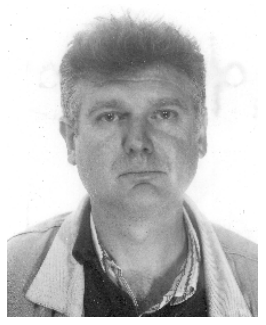

Ricardo Marqués (M'95) was born in San Fernando, Cádiz, Spain, in 1954. He received the Ph.D. degree from the University of Seville, Seville, Spain.

$\mathrm{He}$ is currently an Associate Professor with the Department of Electronics and Electromagnetism, University of Seville. Since 1984, he has been with the Microwave Group of Research, University of Seville, where he developed research on computer-aided design of microwave circuits, wave propagation in ferrites and complex media, and transmission-line theory. More recently, his interest has also focused on the analysis and design of artificial microstructured media with exotic electromagnetic properties (metamaterials) including negative refraction, sub-diffraction imaging, and its applications for microwave technology.

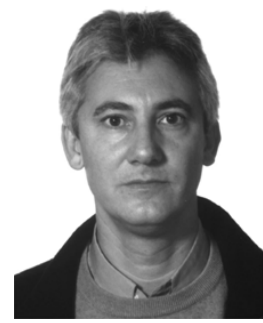

Francisco Medina (M'90-SM'01) was born in Puerto Real, Cádiz, Spain, in November 1960. He received the Licenciado and Doctor degrees from the University of Seville, Seville, Spain, in 1983 and 1987 respectively, both in Physics.

From 1986 to 1987, he spent the academic year with the Laboratoire de Microondes de l'ENSEEIHT, Toulouse, France. From 1985 to 1989, he was an Assistant Professor with the Department of Electronics and Electromagnetism, University of Seville, where, since 1990, he has been an Associate Professor of electromagnetism and is also currently Head of the Microwaves Group. His research interest includes analytical and numerical methods for guiding, resonant, and radiating structures, passive planar circuits, periodic structures, and the influence of anisotropic materials (including microwave ferrites) on such systems. $\mathrm{He}$ is also interested in artificial media modeling and design. He is on the Editorial Board of the International Journal of RF and Microwave Computer-Aided Engineering. He also acts as a reviewer for the Institution of Electrical Engineers (IEE), U.K., and American Physics Association journals.

Dr. Medina was a member of the Technical Programme Committees (TPC) of the 23rd European Microwave Conference, Madrid, Spain, 1993, the ISRAMT'99, Málaga, Spain, 1999, and a member of the TPC of Microwave Symposium, Tetouan, Morocco, 2000. He was co-organizer of the New Trends on Computational Electromagnetics for Open and Boxed Microwave Structures Workshop, Madrid, Spain, 1993. He is a member of the Massachusetts Institute of Technology (MIT) Electromagnetics Academy. He is a reviewer for the IEEE TRANSACTIONS ON MICROWAVE THEORY AND TECHNIQUES. He also acts as reviewer for other IEEE publications. He was the recipient of a 1983 Spanish Ministerio de Educación y Ciencia (MEC) Research Scholarship, a Spanish MEC Scholarship, and a French Ministère de la Recherche et la Technologie Scholarship. 